\title{
Ideación suicida y grupo de iguales: análisis en una muestra de adolescentes venezolanos*
}

\author{
Suicide Ideation and Peers Group: Analysis of \\ a Venezuelan Adolescents Sample
}

Recibido: 23 de julio de 201 | Revisado: 5 de febrero de 2015 | Aceptado: 17 de abril de 2015

\author{
JUAN ANTONIO RODRÍGUEZ RAMÍREZ** \\ JESÚS ÁNGEL ODUBER \\ Universidad de Los Andes, Mérida, Venezuela
}

doi:10.11144/Javeriana.upsy14-3.isgi

Para citar este artículo: Rodríguez, J. A., \& Oduber, J. A. (2015). Ideación suicida y grupo de iguales: análisis en una muestra de adolescentes venezolanos. Universitas Psychologica, 14(3), 1129-1140. http://dx.doi.org/10.11144/Javeriana.upsy14-3.isgi

* Artículo de investigación

** Profesor Asociado. Escuela de Criminología. República Bolivariana de Venezuela. Correos electrónicos: jarodrig@ula.ve, jesusoduber@hotmail. com
RESUMEN

El objetivo de este estudio fue determinar un modelo de relaciones para predecir la ideación suicida de adolescentes escolarizados. Se propuso un modelo basado en la conducta suicida de los iguales, el apoyo del padre y de la madre, la violencia familiar y la depresión. La muestra estuvo conformada por 912 sujetos. Los resultados obtenidos mediante un path analysis indican que los adolescentes con síntomas de depresión optan por amigos con conductas suicidas y estos, además, aumentan directamente la probabilidad de ideación suicida.

Palabras clave

grupo de iguales; familia; depresión; ideación suicida

\footnotetext{
A B S T R A C T

The goal of this study was to determine a model of relationship in order to predict school-age adolescents suicide ideation. A model based on peers' suicidal behavior, father and mother's support, family violence and depression was proposed. A sample of 912 subjects was used. The results through path analysis suggest that teenagers with depression symptoms prefer relating with friends who show suicidal behavior.

Keywords

peers; family; depression; suicide ideation
} 


\section{Introducción}

En Latinoamérica, la tasa de suicidio adolescente varía de un país a otro y las cifras fluctúan de 2.5 a 9.3 casos por cada 100000 habitantes (González, 2007; Toro, Paniagua, González, \& Montoya, 2009; Ventura-Juncá et al., 2010). Asimismo, el contingente de jóvenes que reporta ideas suicidas en esta región puede oscilar entre 16 y 62 \% (Espinoza-Gómez et al., 2010; Florenzano et al., 2011; Gascón, 2005; Ventura-Juncá et al., 2010; Vera \& Díaz, 2012). Es indudable que estas estadísticas evidencian un problema de salud pública, en particular, porque el suicidio se halla a nivel mundial entre las tres principales causas de muerte durante la adolescencia (Organización Mundial de la Salud [OMS], 2014).

Por otra parte, en la generalidad de las investigaciones, el comportamiento suicida -o también llamado espectro suicida- es una escalada de etapas que varía de menor a mayor gravedad (Maris, 2002). Este escalamiento se inicia con la ideación suicida, continúa con la manifestación de amenazas, planes e intentos suicidas y, finalmente, concluye con el suicidio completo o consumado (Viñas, Jané, \& Domènech, 2000). Aunque menos frecuente, otros investigadores propugnan que este proceso no es siempre secuencial y que presentar cualquiera de las conductas suicidas no implica indispensablemente la manifestación de otras (De Wilde, Kienhorst, \& Diekstra, 1996; González, Ramos, Caballero, \& Wagner, 2003). En cualquier caso, lo que sí parece estar mejor establecido es que la ideación suicida se trata de aquellas cogniciones que van desde ideas esporádicas de que la vida no merece vivirla hasta pensamientos de autoaniquilación crónicos y sostenidos (Goldney, Winefield, Tiggemann, Winefield, \& Smith, 1989). Y, además, que es un factor de riesgo tanto del intento suicida como del suicidio consumado; incluso, es el componente más frecuente del espectro suicida y se amplifica su riesgo cuando es crónica (Miranda de la Torre, Cubillas, Román, \& Valdez, 2009).

Del mismo modo, la investigación empírica denota que en la adolescencia concurren varios factores individuales y ambientales que aumentan o reducen la posibilidad de ideación suicida, algunos de ellos son la depresión, la violencia doméstica, el apoyo familiar y el grupo de iguales con conductas suicidas (Espinoza-Gómez et al., 2010; Evans, Hawton, \& Rodham, 2004; Gascón, 2005; Miranda de la Torre et al., 2009; Sánchez-Sosa, Villareal-González, Musitu, \& Martínez, 2010; Srdanović-Maraš, Durić, Marković, \& Biro, 2011; Toro et al., 2009). Respecto a la depresión, en el caso latinoamericano, hay un número substancial de trabajos que señalan una fuerte relación entre este trastorno y la ideación suicida de adolescentes (v. gr., Barroilhet et al., 2012; González et al., 2003; Miranda de la Torre et al., 2009; Toro et al., 2009). En Venezuela, por ejemplo, Gascón (2005) reporta que la ideación suicida de adolescentes escolarizados está influenciada por el sexo, el estrato social, la ansiedad y la depresión. Sin embargo, en la mayoría de estos estudios no queda suficientemente establecido si el efecto de la depresión en el pensamiento suicida es directo o indirecto. Quizá la investigación que ofrece evidencias más sólidas al respecto es la de Sánchez-Sosa et al. (2010) con adolescentes de México. Sus resultados apuntan a que la depresión es una variable que tiene efectos directos en el pensamiento suicida y, de igual forma, actúa como mediadora en la relación entre el funcionamiento familiar, los problemas de integración escolar, la victimización escolar y la variable dependiente ideación suicida.

En cuanto a las variables contextuales o ambientales, el tipo de prácticas parentales está asociado con la ocurrencia de ideación suicida adolescente. En Latinoamérica, por ejemplo, Salvo y Melipillán (2008), mediante un análisis de regresión lineal múltiple, encuentran que la cohesión y el apoyo parental contribuyen a explicar la variabilidad de suicidalidad y, específicamente, son factores que previenen las ideas suicidas de jóvenes chilenos. También en Chile, Florenzano et al. (2011), basándose en un análisis de regresión logística, determinan que la buena relación entre padre y madre, los estilos adecuados de crianza parental y las relaciones convencionales entre padres e hijos evitan el pensamiento suicida de los adolescentes. En México, González et al. (2003), examinando una muestra de adolescentes, detec- 
tan que el afecto y la comunicación de los padres con sus hijos son variables que reducen el riesgo de depresión e ideación suicida, particularmente en varones que reportan una buena relación con sus padres. Igualmente, Sánchez-Sosa et al. (2010) pusieron a prueba un modelo de relaciones para explicar la ideación suicida de adolescentes en ese mismo país, y uno de sus hallazgos principales indica que el efecto del funcionamiento familiar en el pensamiento suicida es indirecto y está mediado por la depresión.

Por su parte, sobre el conflicto y la violencia familiar, González et al. (2003) documentan como correlatos de la ideación suicida en adolescentes mexicanos los problemas del hijo con ambos padres y la discusión de estos por problemas económicos. Espinoza-Gómez y su equipo de investigación (2010) informan acerca de la fuerte asociación entre ideación suicida y percepción de violencia doméstica en todas sus manifestaciones, esto es, violencia psicológica, física y sexual. También en México, Sarmiento y Aguilar (2011) consiguen correlaciones positivas entre las peleas de padres e hijos y el pensamiento suicida de estos. Más aún, el conflicto con la madre es una de las variables predictivas que entra en el modelo obtenido mediante el análisis de regresión lineal múltiple. Además, los trabajos tienden a indicar que la discusión y violencia doméstica aumentan la probabilidad de depresión en adolescentes y, por medio de esta, el riesgo de pensamiento suicida (Espinoza-Gómez et al., 2010; González et al., 2003; Leyva, Hernández, Nava, \& López, 2007; Moad, 2007).

Ahora bien, cabe destacar que las investigaciones en Latinoamérica no han reparado en la manera cómo actúan los amigos que presentan conductas suicidas en la suicidalidad del propio adolescente, pero hay razones para propugnar que el grupo de iguales puede desempeñar un rol significativo en la tendencia suicida de los jóvenes. Quizás el aporte de Sánchez, Guzmán y Cáceres (2005) constituya uno de los pocos estudios sistemáticos en la región que evalúa el impacto de los iguales en la ideación suicida del adolescente. Sus hallazgos apuntan a que los adolescentes con diagnóstico depresivo tienden a imitar las conductas suicidas de sus amigos.
Con referencia a otras contribuciones fuera de Hispanoamérica, Cerel, Roberts y Nilsen (2005), analizando una muestra representativa de 5852 adolescentes, observan que aquellos jóvenes expuestos a amigos con conductas suicidas tienen mayor probabilidad de experimentar ideación e intento suicida y, además, conducta antisocial (v. gr., consumo de alcohol, drogas y agresión). Evans et al. (2004) confirman que varios estudios llegan a conclusiones similares, es decir, hay una asociación significativa entre tener amistades que manifiestan conductas suicidas y el propio pensamiento suicida. En concreto, es el intento suicida de los iguales, y no el suicidio consumado, la variable que ejerce un efecto sobre la ideación suicida de los jóvenes. En contraste, Feigelman y Gorman (2008) determinaron que el suicidio de un amigo está asociado con el pensamiento y la tentativa de suicidio del adolescente y, también, con una mayor depresión durante el primer año del fallecimiento. De igual forma, Gould et al. (2004) sostienen que los adolescentes que reportan la pérdida de un amigo por suicidio, generalmente, presentan a corto plazo un deterioro de su salud mental (v. gr., depresión) y aumenta la incidencia de suicidio consumado; aunque en este estudio no se detalla si esa muerte también incide sobre las cogniciones suicidas del adolescente.

En este sentido, Phillips (1974) reportó que la conducta suicida de los amigos es un correlato de las tendencias suicidas de las personas. Dicho autor interpreta esta correlación de la manera siguiente: 1) los individuos imitan las conductas suicidas de sus iguales o 2) aquellos sujetos con actitudes suicidas buscan entornos con tales propensiones. Agnew (1998) sostiene que el interés del adolescente por el suicidio puede ser el efecto de un proceso de aprendizaje y contacto con familiares o iguales que han manifestado este tipo de conductas, lo cual enseña al joven que el suicidio es una alternativa para solventar problemas. De Wilde (2000) arguye que la conducta suicida manifestada por algún miembro del grupo de iguales del colegio o de fuera de este, puede tener un efecto sobre la suicidalidad del adolescente mediante un proceso sugestivo denominado "contagio suicida". Según Evans et al. (2004), la literatura apunta a que es el proceso 
de modelado de conductas suicidas del grupo de iguales el que ejerce una influencia en la propia tendencia suicida del adolescente. En conclusión, al parecer, los adolescentes cuyo grupo de iguales manifiesta ideas e intentos suicidas y/o suicidios consumados pueden estar en peligro de manifestar comportamientos suicidas.

\section{Objetivos}

El presente estudio es innovador porque robustece los trabajos en español sobre el riesgo de ideación suicida durante la adolescencia. Ante la falta de estudios sistemáticos sobre el tema en la región, se pretende explorar principalmente el papel que juega el grupo de iguales en la probabilidad de ideación suicida; por supuesto, sin exceptuar del análisis el vínculo interactivo de otras variables ligadas a este fenómeno. Tomando en consideración los hallazgos en el área, en este estudio se busca determinar un modelo de relaciones (Figura 1) con el cual se pueda explicar la ideación suicida de adolescentes escolarizados, definiendo el efecto que presenta la conducta suicida de los iguales, la depresión, el apoyo del padre y de la madre y la violencia doméstica.

\section{Método}

\section{Participantes}

El tipo de muestreo en esta investigación fue no probabilístico. De tal manera, la muestra está formada por 912 estudiantes de bachillerato que se encontraban presentes en el aula al momento de aplicar el cuestionario. Los participantes están encuadrados en una franja de edad de 11 a 18 años, cuya media es 14.55 años ( $D E=1.58$ años). Con respecto al sexo, 441 de estos educandos son hombres (48.4\%) y 471 mujeres (51.6\%). Todos los adolescentes formaban parte de cinco centros de enseñanza pública seleccionados por conveniencia en Mérida, Venezuela. La distribución por etapas educativas es la siguiente: 411 sujetos $(45.1 \%)$ formaban parte del ciclo básico (1. $.^{\circ} 2 .^{\circ}$ y $3 .^{\circ}$ año) y 501 (54.9\%) del ciclo diversificado ( $4 .^{\circ}$ y $5 .^{\circ}$ año). Del total de adolescentes escolarizados, un $83.4 \%$

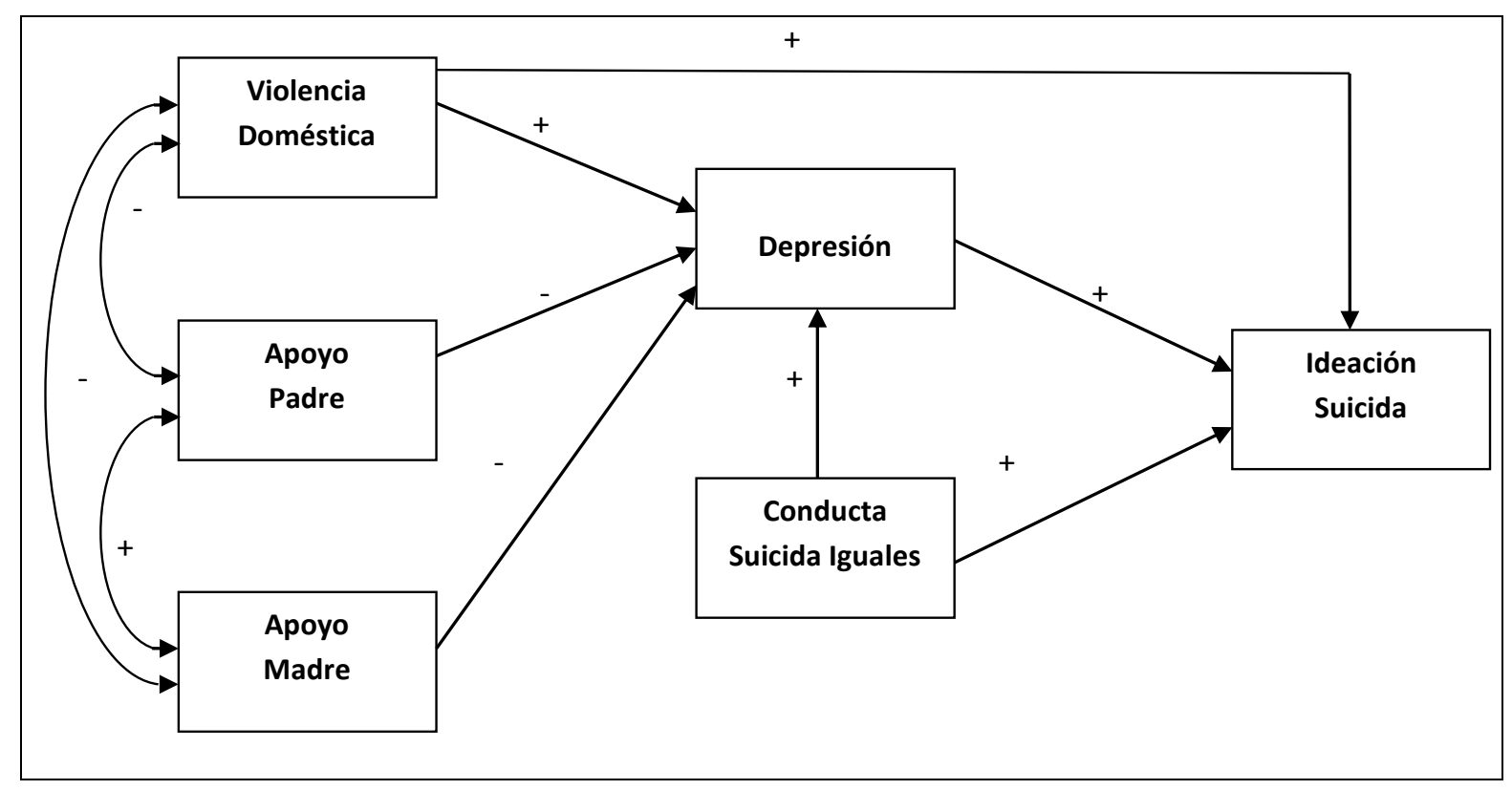

Figura 1. Modelo hipotético de partida sobre ideación suicida.

Fuente: elaboración propia 
reportó que solo estudiaba y el resto (15\%) informó que alternaba los estudios con actividades laborales. Casi la mitad de estos jóvenes pertenecen a familias biparentales (49.7\%), más de un tercio (36.2\%) a familias monoparentales y el resto vive exclusivamente con hermanos u otros familiares (14.1\%).

\section{Instrumentos y variables}

Para la recolección de información se utilizó un conjunto de cuestionarios estandarizados y algunos indicadores ad hoc. Todos han presentado en investigaciones anteriores niveles satisfactorios de confiabilidad y validez (véase con mayor detenimiento a Loinaz, Echeburúa, Ortiz-Tallo, \& Amor, 2012; Rodríguez, Mirón, \& Rial, 2012; Sanz \& GarcíaVera, 2007; Villalobos, 2009, para un análisis tanto del proceso de adaptación de estos cuestionarios al español como lo relativo a su validez y fiabilidad). Las variables y sus respectivos instrumentos se detallan a continuación.

\section{Apoyo parental}

Se usó una adaptación al español del Abusive and Supportive Environments Parenting Inventory (EASE-PI) de Nicholas y Bieber (1997). La subescala de apoyo parental consta de 24 ítems para el caso de los padres (12 ítems referidos a las conductas de afecto del padre y 12 referidos a las conductas de afecto de la madre). Todos estos ítems evalúan la frecuencia con la que cada uno de los progenitores manifiesta conductas encaminadas a ayudar al joven en las dificultades o a proporcionales consuelo y afecto cuando lo necesitan. Cualquiera de estos ítems se responde en un formato Likert de 5 puntos $(0=$ nunca, $1=$ pocas veces, 2 = algunas veces, 3 $=$ bastantes veces y $4=$ con mucha frecuencia). En el presente estudio, el alfa de Cronbach conseguido para el apoyo de la madre fue de 0.92 y para el padre de 0.94 .

\section{Violencia doméstica}

Una adaptación de la Conflict Tactics Scales (CTS) de Straus, Hamby, Boney-McCoy y Sugarman
(1996) se utilizó para evaluar la frecuencia con la que se emplea la violencia física como táctica para resolver conflictos en el hogar (v. gr., "Dar una paliza o golpiza al otro"). El rango de respuesta de los 4 ítems va desde "Nunca" (0) hasta "Siempre" (4). En esta investigación, el alfa de Cronbach observado para la subescala de violencia física fue de 0.86 .

\section{Depresión}

En este estudio se aplicó el Beck Depression Inventory (BDI-IA) (Beck, Steer, Ball, \& Ranieri, 1996) para evaluar la depresión. El inventario de Beck es un autoinforme que se compone de 21 ítems que valoran la gravedad de la depresión. Los 21 factores que miden este cuestionario son: tristeza, pesimismo, sensación de fracaso, insatisfacción, culpa, sentimiento de castigo, autodesprecio, autoacusación, ideas de suicidio, llanto, irritabilidad, desinterés social, indecisión, cambios en la imagen corporal, dificultad para trabajar, insomnio, fatiga, pérdida de apetito, pérdida de peso, preocupaciones somáticas y bajo nivel de libido. Cada ítem presenta cuatro categorías de respuesta cuyo rango oscila entre 0 y 3 puntos. Debido a los objetivos de esta investigación, se excluyó del sumatorio el factor sobre ideas suicidas. El alfa de Cronbach obtenido en este estudio fue de 0.87 .

\section{Conducta suicida de los iguales}

Sobre la base de la literatura en el área (v. gr., Prinstein, Boergers, \& Spirito, 2001), se elaboraron tres ítems referidos a la ocurrencia de pensamiento suicida, intento suicida y suicidio consumado de los iguales. Particularmente, se les preguntó a los adolescentes sobre las siguientes situaciones: 1) ¿Cuántos de tus amigos piensan que el suicidio es la única vía de escape ante los problemas de su vida? 2) ¿Cuántos de tus amigos han intentado suicidarse en este último año? y 3) ¿Cuántos de tus amigos se han suicidado en este último año? La categoría de respuesta de estas preguntas se establece en una escala Likert de cuatro puntos $(0=$ ninguno, $1=$ algunos, $2=$ casi todos y $3=$ todos). Se estimó un índice global totalizando los 3 ítems. Esta variable 
se ha denominado conducta suicida de los iguales. El alfa de Cronbach conseguido fue de 0.72 .

\section{Ideación suicida}

El Positive and Negative Suicide Ideation Inventory (PANSI) de Osman, Gutiérrez, Kopper, Barrios y Chiros (1998) fue el cuestionario administrado para evaluar la ideación suicida de los adolescentes. Una versión en español fue validada por Villalobos (2009) en Colombia y es la que forma parte del presente trabajo. El instrumento original y la adaptación en español incluyen un total de 14 ítems en los cuales subyacen dos factores. Uno de estos factores se relaciona con la ideación suicida positiva y consta de 6 ítems, el otro se refiere a la ideación suicida negativa y suma en total 8 ítems. A la persona encuestada se le pregunta sobre la frecuencia con la que ha presentado pensamientos suicidas durante el último año. La categoría de respuesta está formulada en una escala Likert de 5 puntos que varía en un intervalo de 0 (nunca) a 4 (siempre). La fiabilidad estimada por medio del alfa de Cronbach fue de 0.87 en este estudio.

\section{Procedimiento}

Luego de solicitar y obtener la autorización de los planteles educativos, los instrumentos fueron aplicados de forma colectiva en el aula donde se desarrollan las actividades académicas. Al comienzo, se les explicó a los participantes el propósito de la investigación y se insistió en el anonimato de las respuestas y, además, de su participación voluntaria en el levantamiento de información. La muestra se tomó en noviembre de 2012. De un total de 1 187 cuestionarios aplicados, se eliminaron 275 por inconsistencias y errores en la información suministrada.

\section{Análisis de los datos}

Para fijar las características generales de la muestra en cuanto a las variables de estudio, se analizaron las principales medidas de centralidad y dispersión, esto es, media, desviación estándar y rangos. Tam- bién para explorar la asociación entre cada uno de los factores analizados y la ideación suicida, se llevó a cabo un análisis de correlación de Pearson. Ambos análisis se efectuaron por medio del programa estadístico SPSS (versión 17.0). A continuación, mediante el programa AMOS 18.0 (Arbuckle, 2009), se desarrolló un análisis de senderos (path analysis) con el objeto de poner a prueba el modelo hipotético descrito en la Figura 1. En este caso, se escogió el Método de Máxima Verosimilitud para la estimación de parámetros. Así, se revisaron los índices de bondad de ajuste entre el modelo inicial y los datos empíricos de la muestra para observar su idoneidad. En concreto, se verificó tanto el contraste sobre la distribución $\chi^{2}$ como el resto de índices de bondad de ajuste. Según Hair, Anderson, Tatham y Black (2000) un modelo propuesto presenta un buen ajuste a los datos observados cuando el producto entre el $\chi^{2}$ y los grados de libertad es menor a 3, los índices de ajuste NFI, CFI y GFI son iguales o superiores a 0.9 y el RMSEA es menor a 0.08. Finalmente, por los problemas de ajuste hallados, se reespecificó el modelo de partida sobre la base de los criterios siguientes: 1) los niveles de significación de los parámetros (relaciones entre variables) estimados, 2) la información proporcionada por la matriz residual y 3) los índices de modificación establecidos por el propio programa AMOS.

\section{Resultados}

\section{Descriptivos y correlaciones}

Según la Tabla 1, los niveles percibidos de apoyo parental son, en promedio, altos y es poco frecuente la violencia física en los hogares de estos jóvenes. Por término medio, estos adolescentes interactúan con muy pocos amigos que manifiestan conductas suicidas y, a la vez, reportan bajos niveles de depresión e ideación suicida. Sin embargo, es conveniente contrastar esta tendencia con la variabilidad presente en algunas puntuaciones, en especial, la que confirman la desviación estándar y el rango observado. Simultáneamente, se puede comprobar con el análisis de correlación de la Tabla 1, que el apoyo del padre y de la madre disminuye la probabilidad 
TABLA 1

Coeficientes de correlación de Pearson, media, desviación estándar y rangos de las variables observadas

\begin{tabular}{|c|c|c|c|c|c|c|}
\hline & 1 & 2 & 3 & 4 & 5 & 6 \\
\hline 1. Apoyo madre & - & & & & & \\
\hline 2. Apoyo padre & $0.55 * * *$ & - & & & & \\
\hline 3. Violencia doméstica & $-0.23 * * *$ & $-0.24 * * *$ & - & & & \\
\hline 4. Conducta suicida iguales & $-0.13 * * *$ & $-0.14 * * *$ & $0.25 * * *$ & - & & \\
\hline 5. Depresión & $-0.27 * * *$ & $-0.32 * * *$ & $0.27 * * *$ & $0.31 * * *$ & - & \\
\hline 6. Ideación suicida & $-0.28 * * *$ & $-0.27 * * *$ & $0.31 * * *$ & $0.29 * * *$ & $0.53 * * *$ & - \\
\hline Media & 36.68 & 31.46 & 1.21 & 0.65 & 5.36 & 8.91 \\
\hline$(\mathrm{DE})$ & $(10.2)$ & $(13.03)$ & $(2.68)$ & $(1.15)$ & $(6.72)$ & $(8.71)$ \\
\hline Rango teórico & $0-48$ & $0-48$ & $0-16$ & $0-9$ & $0-60$ & 0.56 \\
\hline Rango observado & $0-47$ & $0-47$ & $0-16$ & $0-9$ & 0.59 & 0.52 \\
\hline
\end{tabular}

Fuente: elaboración propia

de ideación suicida del adolescente y, al contrario, la violencia doméstica, el comportamiento suicida de los iguales y la depresión la aumentan.

\section{Análisis de Senderos (Path Analysis)}

Coherente con la estrategia de análisis de datos, se puso a prueba el modelo planteado en la Figura 1. En la Tabla 2, se observa que los índices de bondad de ajuste entre el modelo inicial y los datos empíricos no fueron enteramente apropiados. Especialmente, el producto entre $\chi^{2} / g l$ y el RMSEA revelan problemas de ajuste del modelo propuesto tal como lo señalan Hair et al. (2000). Esto hizo necesario reespecificar el modelo hipotético de partida según los criterios descritos en párrafos anteriores. El proceso de reespecificación se desarrolló paso a paso, hasta lograr un modelo cuyo ajuste a los datos empíricos fuera apropiado. En consecuencia, se obtuvo un modelo final, cuyos índices de ajuste se resumen también en la Tabla 2 . De acuerdo a esta tabla, los diferentes índices analizados presentan un mejoramiento en la calidad de ajuste.

El modelo final de relaciones entre las variables queda entonces configurado según los resultados presentados en la Figura 2. En cuanto a esta representación gráfica, se observa que hay una correlación positiva y significativa entre el apoyo del padre y el apoyo de la madre $(r=0.56, p<0.001)$ y una correlación negativa de estas dos variables con la violencia doméstica $(r=-0.2, p<0.001$ en ambos casos).

Con respecto a los efectos directos, en el modelo final se observa que la depresión presenta un efecto importante en la ideación suicida $(\beta=0.5, p<$ 0.001). En concreto, altos niveles de depresión están asociados a altos niveles de pensamiento suicida. De igual forma, la conducta suicida de los iguales tiene un efecto significativo en la variable explicada ( $\beta$ $=0.11, p<0.01)$. Así, cuanto mayor sea el contacto con amigos que manifiestan comportamientos suicidas (v. gr., pensamientos e intentos suicidas

TABLA 2

Índices de bondad de ajuste del modelo inicial y el modelo final

\begin{tabular}{lcccccccc}
\hline & $\chi^{2}$ & $\mathrm{gl}$ & $\mathrm{p}$ & $\chi^{2} / \mathrm{gl}$ & NFI & CFI & GFI & RMSEA \\
\hline Modelo Inicial & 30.9 & 3 & 0 & 10.29 & 0.93 & 0.94 & 0.97 & 0.16 \\
\hline Modelo Final & 3.2 & 4 & 0.52 & 0.8 & 0.99 & 1 & 0.99 & 0.01 \\
\hline
\end{tabular}

Fuente: elaboración propia 


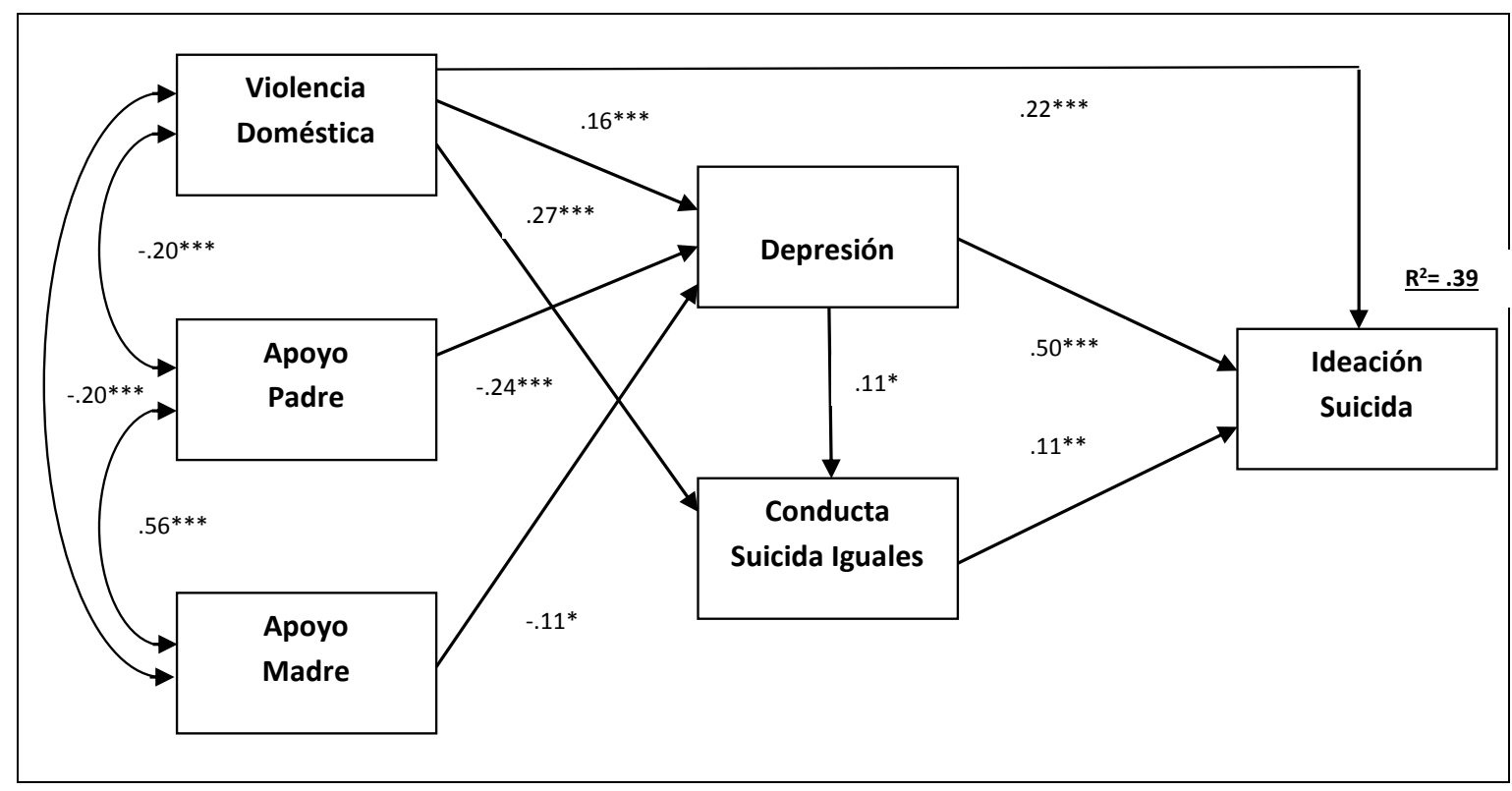

Figura 2. Modelo final de relaciones sobre ideación suicida $(n=369)$.

$* * * p<0.001 * * p<0.01 * p<0.05$

Fuente: elaboración propia

y suicidio consumado) mayor la probabilidad de ideas suicidas en los adolescentes. Por último, otro efecto directo es el de la violencia doméstica en la ideación suicida $(\beta=0.22, p<0.001)$. El percibir violencia física en el hogar aumenta directamente el pensamiento suicida de los jóvenes.

Además de estos, en el modelo final se observan otros efectos indirectos de especial consideración. De tal manera, el apoyo de la madre y del padre presentan una relación negativa y significativa con la depresión $(\beta=-0.11, p<0.05$ y $\beta=-0.24, p<$ 0.001 , respectivamente) y esta última con la ideación suicida. La violencia doméstica tiene un efecto indirecto en la ideación suicida, mediante su relación positiva con la depresión $(\beta=0.16, p<0.001)$. Asimismo, la violencia doméstica y la depresión también muestran una relación indirecta con la ideación suicida por medio de su efecto en la conducta suicida de los iguales $(\beta=0.27, p<0.001$ y $\beta=0.11, p<$ 0.05 , respectivamente). El efecto indirecto de ambas variables fue indicado por la propia técnica de análisis. Por último, el modelo obtenido explica el 39 \% de la varianza de la ideación suicida.

\section{Discusión y conclusión}

Como ya se explicó, el objetivo principal de este estudio ha sido poner a prueba un modelo estructural que incluya la conducta suicida de los iguales, para estimar su influencia en la ideación suicida adolescente. Ante la pregunta sobre cuáles son los factores que amplifican o minimizan el pensamiento suicida de los jóvenes, algunos modelos explicativos han fusionado factores individuales relacionados con eventos de vida estresantes, desesperanza, depresión, autoestima, solución de problemas, consumo de drogas, soledad, autoeficacia, etc., con factores contextuales referidos, por ejemplo, al conflicto familiar, cohesión familiar, problemas económicos en la familia, abuso físico paterno, divorcio, apoyo de los profesores, apoyo social, etc. (v. gr., SánchezSosa et al., 2010; Villalobos, 2009). Sin embargo, en la mayoría de estos modelos no se incluye al grupo de iguales como variable explicativa que, indiscutiblemente, juega un rol fundamental en la adolescencia y, desde luego, en el ajuste psicosocial del joven. 
Con base en lo anterior, el modelo final de este estudio indica que el grupo de iguales con conductas suicidas tiene un efecto directo en la ideación suicida del adolescente. Si bien no se encontró un hallazgo similar en otras publicaciones que han aplicado como técnica de análisis las Ecuaciones Estructurales, este dato concuerda con trabajos que consiguen a nivel bivariado y multivariante que la suicidalidad del grupo de iguales es un predictor del pensamiento suicida de los adolescentes (v. gr., Evans et al., 2004; Feigelman \& Gorman, 2008; Prinstein et al., 2001). Por supuesto, esta evidencia empírica puede interpretarse de varias maneras. Por ejemplo, que los adolescentes aprenden en sus grupos de iguales con inclinaciones suicidas definiciones, racionalizaciones y motivos favorables al suicidio. Cabe decir, además, que en un entorno grupal con tales características los mecanismos de aprendizaje que facilitan el pensamiento suicida son los que se han propuesto generalmente en psicología, es decir, los de reforzamiento y modelado. Esta propuesta explicativa es coherente con las interpretaciones hechas por Phillips (1974), Agnew (1998) y Evans et al. (2004). Sin embargo, también el efecto directo que genera la suicidalidad de los iguales sobre la propia ideación suicida puede ser explicado por otros procesos grupales como, por ejemplo, el "contagio social" visto como un mecanismo de sugestión (De Wilde, 2000). En cualquier caso, los hallazgos de esta investigación confirman que la conducta suicida de los iguales tiene un efecto directo sobre la probabilidad de pensamiento suicida, y al ser la adolescencia una etapa marcada por la constante interacción e influencia de los iguales, esta característica debe ser considerada en cualquier intento de predicción y explicación del comportamiento suicida durante esta etapa del desarrollo.

También, desde un punto de vista grupal, en el modelo inicial de partida se propuso un efecto indirecto del grupo de iguales sobre la variable dependiente mediante la depresión. Esta relación se planteó sobre la base de los hallazgos develados por Feigelman y Gorman (2008) los cuales apuntan a que la suicidalidad de un amigo aumenta la depresión y el pensamiento suicida del adolescente. Sin embargo, el path analysis indicó que tal relación, al menos en esta muestra, no es empíricamente plausible; más bien al contrario, los índices de modificación del programa AMOS 18 demuestran que la relación es inversa, es decir, presentar síntomas depresivos aumenta la posibilidad de asociarse con amigos que manifiestan tendencias suicidas. Provisionalmente, este efecto permite inferir que la depresión instiga a los adolescentes a buscar grupos de iguales con comportamientos inadecuados, en este caso, otros jóvenes que experimentan ideas e intentos suicidas o, incluso, amistades que terminan quitándose la vida.

Sumado a esto, el modelo estructural permite determinar que el apoyo tanto de la madre como del padre son variables que afectan indirectamente la ideación suicida, mediante su incidencia en la depresión. Este hallazgo es coherente con el reportado por otros autores como Sánchez-Sosa et al. (2010) y Srdanović-Maraš et al. (2011). También, desde el propio dominio familiar, la percepción de violencia doméstica aumenta directamente el pensamiento suicida del adolescente, lo cual coincide con los resultados de otros autores que destacan el papel estresante de esta variable en las ideas suicidas ( $\mathrm{v}$. gr., Espinoza-Gómez et al., 2010). Igualmente, los jóvenes que presencian abusos físicos en sus hogares experimentan depresión, lo que subsiguientemente tiene un efecto en las ideas suicidas. Es más, percibir problemas de maltrato doméstico incrementa el riesgo de relacionarse con grupos que manifiestan problemas de suicidalidad y, por medio de estos, el riesgo de ideación suicida. Aunque este efecto indirecto no fue establecido en el modelo de partida, el mismo fue indicado por el análisis de senderos. Es evidente entonces que un entorno familiar disfuncional, caracterizado por un bajo apoyo y por el uso de la violencia física, tiene una influencia tanto en la aparición de trastornos mentales como en la toma de contacto con un grupo de amigos con conductas inadecuadas, lo que en conjunto aumenta la posibilidad de pensamiento suicida.

Sobre la depresión, el modelo deja claro que es la variable clave y, sin duda, la que mejor explica la ideación suicida. La fuerza de tal efecto, en este caso directo, coincide con los hallazgos de otras investigaciones (v. gr., Konick \& Gutiérrez 2005; Sánchez- 
Sosa et al., 2010). Asimismo, la propiedad mediadora y los posibles efectos indirectos que presenta este trastorno en el modelo final, permiten confirmar su destacado papel en la etiología del fenómeno abordado. Por ende, y congruente con lo señalado por Konick y Gutiérrez (2005), la depresión es una variable individual que no debe ser excluida de ningún modelo explicativo de ideación suicida en la adolescencia; sobre todo, si está demostrado empíricamente que el $60 \%$ de los varones y el $44 \%$ de las mujeres que contemplan el suicidio y que han intentado quitarse la vida, presentan síntomas de depresión moderada y grave (Srdanović-Maraš et al., 2011). Redondeando estas observaciones, los adolescentes deprimidos que piensan en suicidarse presentan un contexto multisistémico que incluye un hogar con problemas de afecto, conflicto y violencia física, además engloba amistades tendentes a la suicidalidad, sin descartar que también formen parte otros factores tales como el fracaso escolar, los trastornos alimenticios, la impulsividad o la conducta antisocial (Sánchez-Sosa et al., 2010).

Con base en lo anterior, hay que aclarar que no es plausible apoyar o rechazar el orden de causalidad de las variables observadas, dado el carácter transversal de este estudio; sin embargo, las relaciones propuestas en el modelo provienen de la literatura en el área. No es superfluo indicar que estas relaciones deben comprenderse con discreción.

Finalmente, de cara al futuro, se propone replicar este modelo estructural en otros estudios transversales considerando que, de ser posible, puede tener más valor probar los efectos aquí encontrados y explicados mediante estudios longitudinales. También puede resultar una valiosa contribución contrastar separadamente el efecto de los tres tipos de conductas suicidas de los iguales (ideación, intento y consumación), de tal manera que se consiga precisar cuál tiene una mayor capacidad explicativa del pensamiento suicida adolescente. Igualmente, sería adecuado probar el poder explicativo y la invariabilidad de estos modelos con otros grupos que puedan estar conformados por categorías ligadas al género, edad o, incluso, a adolescentes de la población general y/o clínica. Analizar diferentes modelos en estos términos, puede guiar la elaboración de programas de intervención mucho más completos dirigidos a la prevención de la ideación suicida.

\section{Referencias}

Agnew, R. (1998). The approval of suicide: A socialpsychological model. Suicide and Suicide and Life-Threatening Behavior, 28(2) 205-225. doi: 10.1111/j.1943-278X.1998.tb00640.x

Arbuckle, J. L. (2009). Amos 18 user`s guide. Chicago: Amos Development Corporation.

Barroilhet, S., Fritsch, R., Guajardo, V., Martínez, V., Vöhringer, P., Araya, R., \& Rojas, G. (2012). Ideas autolíticas, violencia autoinfligida y síntomas depresivos en escolares chilenos. Revista Médica de Chile, 140(7), 873-881. Recuperado de http://www. scielo.cl/pdf/rmc/v140n7/art07.pdf

Beck, A., Steer, R., Ball, R., \& Ranieri, W. (1996). Comparison of Beck Depression Inventary-IA and II in psychiatric outpatients. Journal of Personality Assessment, 67, 588-597. doi: 10.1207/ s15327752jpa6703_13

Cerel, J., Roberts, T., \& Nilsen, W. (2005). Peer suicidal behavior and adolescents risk behavior. Journal of Nervous and Mental Disease, 193(4), 237-243.

De Wilde, E. (2000). Adolescent suicidal behavior: A general population perspective. En K. Hawton \& K. Van Heeringen (Eds.), The international handbook of suicide and attempted suicide (pp. 249-259). Chichester: Wiley.

De Wilde, E., Kienhorst, I., \& Diekstra, R. (1996). El suicidio en la adolescencia. En J. Buendía (Ed.), Psicopatología en niños y adolescentes. Desarrollos actuales (pp. 309-332). Madrid: Pirámide.

Espinoza-Gómez, F., Zepeda-Pamplona, V., BautistaHernández, V., Hernández-Suarez, C., NewtonSánchez, O., \& Plasencia-García, G. (2010). Violencia doméstica y riesgo de conducta suicida en universitarios adolescentes. Salud Pública de México, 52 (3), 213-219. Recuperado de http//www. scielo.org.mx/pdf/spm/v52n3/05.pdf

Evans, E., Hawton, K., \& Rodham, K. (2004). Factors associated with suicidal phenomena in adolescents: A systematic review of population-based studies. Clinical Psychology Review, 24, 957-979. doi: 10.1016/j.cpr.2004.04.005 
Feigelman, W., \& Gorman, B. (2008). Assessing the effects of peer suicide on youth suicide. Suicide and Life-Threatening Behavior, 38(2), 181-194. doi: 10.1521/suli.2008.38.2.181

Florenzano, R., Valdés, M., Cáceres, E., Santander, S., Aspillaga, C., \& Musalem, C. (2011). Relación entre ideación suicida y estilos parentales en un grupo de adolescentes chilenos. Revista Médica de Chile, 139, 1529-1533. Recuperado de http://www. scielo.cl/pdf/rmc/v139n12/art01.pdf

Gascón, A. I. (2005). Ideación suicida, humor depresivo y ansiedad en la adolescencia intermedia escolarizada de la Parroquia Antonio Spinetti Dini: algunos factores de riesgo. Mérida 2005 (Tesis de especialización en Psiquiatría). Universidad de Los Andes, Mérida, Venezuela. Disponible en http:/tesis.ula.ve/postgrado/tde_busca/arquivo.php?codArquivo=2948

Goldney, R., Winefield, A., Tiggemann, M., Winefield, H., \& Smith, S. (1989). Suicidal ideation in a young adult population. Acta Psychiatrica Scandinavica, 79, 481-489. doi: 10.1111/j.1600-0447.1989. tb10291.x

González, J. (2007). Epidemiología de los suicidios. Colombia, 2007. En Forensis 2007. Datos para la vida (pp. 179-204). Bogotá: Instituto Nacional de Medicina Legal y Ciencias Forenses.

González, C., Ramos, L., Caballero, M., \& Wagner, F. (2003). Correlatos psicosociales de depresión, ideación e intento suicida en adolescentes mexicanos. Psicothema, 15(4), 524-532. Recuperado de http// www.psicothema.com/pdf/1102.pdf

Gould, M., Drew, V., Kleinman, M., Lucas, C., Thomas, J., \& Chung, M. (2004). Teenagers'attitudes about coping strategies and help-seeking for suicidality. Journal of the American Academy of Child and Adolescents Psychiatry, 43, 1124-1133. doi: 10.1097/01. chi.0000132811.06547.31

Hair, J., Anderson, R., Tatham, R., \& Black, W.C. (2000). Análisis multivariante. Madrid: Prentice Hall.

Konick, L., \& Gutiérrez, P. (2005). Testing a model of suicide ideation in college students. Suicide and Life-Threatening Behavior, 35, 181-192. doi: 10.1521/ suli.35.2.181.62875

Leyva, J., Hernández, J., Nava, J., \& López, G. (2007). Depresión en adolescentes y funcionamiento fa- miliar. Revista Médica del Instituto Mexicano de Seguro Social, 45 (3), 225-232. Recuperado de http:// new.medigraphic.com/cgi-bin/contenido.cgi?IDR EVISTA $=46 \&$ IDPUBLICACION $=1295$

Loinaz, I., Echeburúa, E., Ortiz-Tallo, M., \& Amor, P. (2012). Propiedades psicométricas de las Conflict Tactics Scales (CTS-2) en una muestra española de agresores de pareja. Psicothema, 24(1), 142 148. Recuperado de http://www.psicothema.com/ pdf/3991.pdf

Maris, R. W. (2002). Suicide. The Lancet, 360(9329), 319-326.

Miranda de la Torre, I., Cubillas, M., Román, R., \& Valdez, E. (2009). Ideación suicida en población escolarizada infantil: factores psicológicos asociados. Salud Mental, 32(6), 495-502. Recuperado de http://www.redalyc.org/pdf/582/58122267007.pdf

Moad, C. (2007). Depresión en adolescentes de escuelas públicas y privadas. Psicología Iberoamericana, 15(1), 6-12. Recuperado de http://www.redalyc. org/articulo.oa?id=133915928002

Nicholas, K., \& Bieber, S. (1997). Assessment of perceived parenting behaviors: The Exposure to Abusive and Supportive Environments Parenting Inventory (EASE-PI). Journal of Family Violence, 12(3), 275-291.

Organización Mundial de la Salud. (2014). Saludpara los adolescentes del mundo. Una segunda oportunidad en la segunda década. Ginebra: Autor. Recuperado de http//apps.who.int/adolescents/second-decade/ files/WHO_FWC_MCA_14.05_spa.pdf

Osman, A., Gutiérrez, P., Kopper, B., Barrios, F., \& Chiros, C. (1998). The positive and negative Suicide Ideation Inventory: Development and validation. Psychological Reports, 82, 783-793. doi: 10.2466/ pr0.1998.82.3.783

Phillips, D. (1974). The influence of suggestion on suicide: Substantive and theoretical implications of the Werther effects. American Sociological Review, 39, 340-354.

Prinstein, M., Boergers, J., \& Spirito, A. (2001). Adolescents'and their friends'health-risk behaviour: Factors that alter or add to peer influence. Journal of Pediatric Psychology, 26(5), 287-298. doi: 10.1093/jpepsy/26.5.287 
Rodríguez, J. A., Mirón, L., \& Rial, A. (2012). Análisis de la relación entre grupo de iguales, vinculación familiar y escolar, autocontrol y conducta antisocial, en una muestra de adolescentes venezolanos. Revista de Psicología Social, 27(1), 25-38. doi: 10.1174/021347412798844033

Salvo, L., \& Melipillán, R. (2008). Predictores de la suicidalidad en adolescentes. Revista Chilena de Neuro-Psiquiatría, 46(2), 115-123. Recuperado de http://www.scielo.cl/pdf/rchnp/v46n2/art05.pdf

Sánchez, R., Guzmán, Y., \& Cáceres, H. (2005). Estudio de la imitación como factor de riesgo para ideación suicida en estudiantes universitarios adolescentes. Revista Colombiana de Psiquiatría, 34(1), 12-25. Recuperado de http//www.redalyc. org/pdf/806/80628403003.pdf

Sanz, J., \& García-Vera, M. (2007). Análisis psicométrico de las versiones breves del Inventario para la Depresión de Beck de 1978 (BDI-IA). Psicología Conductual, 15(2), 191-214. Recuperado de http// www.funveca.org/revista/pedidos/product.php?id_ product $=288$

Sánchez-Sosa, J., Villareal-González, M., Musitu, G., \& Martínez, B. (2010). Ideación suicida en adolescentes: un análisis psicosocial. Intervención Psicosocial, 19(3), 279-287. doi: 10.5093/in2010v19n3a8

Sarmiento, C., \& Aguilar, J. (2011). Predictores familiares y personales de la ideación suicida adolescentes. Psicología y Salud, 21(1), 25-30. Recuperado de http://www.uv.mx/psicysalud/psicysalud-21-1/21-1/ Carolina-Sarmiento-Silva.pdf

Srdanović-Maraš, J., Durić, O., Marković, J., \& Biro, M. (2011). Family and individual factors of suicidal ideation in adolescents. PSIHOLOGIJA, 44(3), 245-260. doi: 10.2298/PSI1103245S

Straus, M., Hamby, S., Boney-McCoy, S., \& Sugarman, D. (1996). The revised Conflict Tactic Scales. Development and preliminary psychometric data. Journal of Family Issues, 17(3), 283-316. Recuperado de http://pubpages.unh.edu/ mas2/CTS15.pdf

Toro, D., Paniagua, R., González, C., \& Montoya, B. (2009). Caracterización de adolescentes escolarizados con riesgo de suicidio, Medellín, 2006. Revista Salud Pública, 27(3), 302-308. Recuperado de http://www.scielo.org.co/pdf/rfnsp/v27n3/ v27n3a07.pdf

Ventura-Juncá, R., Carvajal, C., Undurraga, S., Vicuña, P., Egaña, J., \& Garib, M. (2010). Prevalencia de ideación e intento suicida en adolescentes de la Región Metropolitana de Santiago de Chile. Revista Médica de Chile, 138, 309-315. Recuperado de http://www.scielo.cl/pdf/rmc/v138n3/art08.pdf Vera, Ó., \& Díaz, C. (2012). Suicidio en adolescentes de Sudamérica: un problema creciente. Salud Pública de México, 54(4), 363-364. Recuperado de http:// www.scielosp.org/pdf/spm/v54n4/03.pdf

Villalobos, F. (2009). Ideación suicida en jóvenes: formulación y validación del modelo integrador explicativo en estudiantes de educación secundaria y superior (Tesis Doctoral). Universidad de Granada, España. Recuperado de http://hera.urg.es/ tesisugr/1805982x.pdf

Viñas, F., Jané, M., \& Domènech, E. (2000). Evaluación de la severidad de la ideación suicida autoinformada en escolares de 8 a 12 años. Psicothema, 12(4), 594-598. Recuperado de http://www.psicothema. com/pdf/377.pdf 